\title{
Clinician Productivity During Student Full Time Clinical Experiences
}

Jamie Dehan

University of Saint Mary, jamie.dehan@stmary.edu

Michele Avery

Kansas City Kansas Community College, micheleavery1@yahoo.com

Trevor Elmer

University of Kansas Medical Center, telmer5592@gmail.com

Yvonne Colgrove

University of Kansas Medical Center, ycolgrove@kumc.edu

Follow this and additional works at: https://nsuworks.nova.edu/ijahsp

Part of the Interprofessional Education Commons, and the Physical Therapy Commons

\section{Recommended Citation}

Dehan J, Avery M, Elmer T, Colgrove Y. Clinician Productivity During Student Full Time Clinical Experiences. The Internet Journal of Allied Health Sciences and Practice. 2021 Oct 01;19(4), Article 6.

This Manuscript is brought to you for free and open access by the College of Health Care Sciences at NSUWorks. It has been accepted for inclusion in Internet Journal of Allied Health Sciences and Practice by an authorized editor of NSUWorks. For more information, please contact nsuworks@nova.edu. 


\title{
Clinician Productivity During Student Full Time Clinical Experiences
}

\begin{abstract}
Purpose: Clinical education is an essential part of physical therapy education. Clinicians who provide student clinical training have the primary responsibility of patient care and meeting their job demands including meeting productivity standards. Adding a student to the mix is often perceived as negatively impacting productivity. The purpose of this study is to examine the impact on clinician productivity in direct patient care while hosting a student for a clinical experience. This study (a) investigates differences seen when hosting a DPT student or a PTA student; (b) compares differences seen influenced by which curricular stage the student is in for the clinical experience; and (c) investigates the impact on productivity while hosting a student across various clinic settings. Method: Clinical instructors for DPT and PTA students from three academic institutions were asked to participate while hosting one of the institution's students. Information was taken on daily hours worked, number of patients seen, and units billed for the week before the student arrived and during the student's full-time clinical experience. Data collected was analyzed for changes over time and for comparison of baseline data to collective (net) averages for all weeks in which the student was present. Sub-analysis was done on curricular timing of the rotations (initial, intermediate, or final), types of settings (inpatient, outpatient or other), and types of students. Results: While there were several significant changes over time in the components assessed, there were no significant differences when comparing baseline productivity components to the collective weekly averages. Conclusion: It appears that hosting students during full-time clinical experiences does not affect the overall productivity. There is generally the predictable drop initially that recovers to baseline around week 4 while either maintaining or increasing productivity during the remaining weeks to produce a net neutral or positive impact. This study works to ultimately disprove the common belief that clinician productivity is negatively impacted while hosting a student during a clinical experience.
\end{abstract}

\section{Author Bio(s)}

Jamie Dehan, PT, PhD, is an Associate Professor at the University of Saint Mary in Leavenworth, KS. She also serves as the Stefani Doctor of Physical Therapy Program Director and former Director of Clinical Education. She is a licensed physical therapist in the states of Kansas and Missouri.

Michele Avery, PTA, MS, is a tenured faculty member and serves as the Program Director and Academic Coordinate of Clinical Education for the Physical Therapist Assistant Program at Kansas City Kansas Community College, Kansas City, KS. She is a licensed physical therapist assistant in the states of Kansas and Missouri.

Trevor Elmer, PT, was a student in the Doctor of Physical Therapy Program at the University of Kansas Medical Center in Kansas City, KS. He now works as a licensed physical therapist in an outpatient clinic in Colorado.

Yvonne Colgrove, PT, PhD, is an Associate Clinical Professor at the University of Kansas Medical Center in Kansas City, KS. She serves as the Director of Clinical Education in the Doctor of Physical Therapy Program. She is a founding member of the LEARN-PT Lab.

\section{Acknowledgements}

Thank you to all the clinical instructors and students that participated in this study. 


\title{
1IJAHSP \\ The Internet Joumnal of Allied Health Sciences and Practice \\ Dedicated to allied health professional practice and education \\ Vol. 19 No. 4 ISSN 1540-580X
}

\section{Clinician Productivity During Student Full Time Clinical Experiences}

\author{
Jamie Dehan \\ Michele Avery \\ Trevor Elmer \\ Yvonne Colgrove
}

1. University of Saint Mary

2. Kansas City Kansas Community College

3. University of Kansas Medical Center

United States

\begin{abstract}
Purpose: Clinical education is an essential part of physical therapy education. Clinicians who provide student clinical training have the primary responsibility of patient care and meeting their job demands including meeting productivity standards. Adding a student to the mix is often perceived as negatively impacting productivity. The purpose of this study is to examine the impact on clinician productivity in direct patient care while hosting a student for a clinical experience. This study (a) investigates differences seen when hosting a DPT student or a PTA student; (b) compares differences seen influenced by which curricular stage the student is in for the clinical experience; and (c) investigates the impact on productivity while hosting a student across various clinic settings. Method: Clinical instructors for DPT and PTA students from three academic institutions were asked to participate while hosting one of the institution's students. Information was taken on daily hours worked, number of patients seen, and units billed for the week before the student arrived and during the student's full-time clinical experience. Data collected was analyzed for changes over time and for comparison of baseline data to collective (net) averages for all weeks in which the student was present. Subanalysis was done on curricular timing of the rotations (initial, intermediate, or final), types of settings (inpatient, outpatient or other), and types of students. Results: While there were several significant changes over time in the components assessed, there were no significant differences when comparing baseline productivity components to the collective weekly averages. Conclusion: It appears that hosting students during full-time clinical experiences does not affect the overall productivity. There is generally the predictable drop initially that recovers to baseline around week 4 while either maintaining or increasing productivity during the remaining weeks to produce a net neutral or positive impact. This study works to ultimately disprove the common belief that clinician productivity is negatively impacted while hosting a student during a clinical experience.
\end{abstract}

Keywords: clinical education, productivity, DPT student, PTA student, clinical settings 


\section{INTRODUCTION}

Clinical education is an essential part of student learning and training for developing competency in entry level practice. ${ }^{1}$ On average, 29 percent of the curriculum in doctoral physical therapist (DPT) education programs in the US is devoted to full-time clinical education experiences. ${ }^{2}$ Similarly, on average, 19 percent of the curriculum in physical therapist assistant (PTA) education programs in the US is devoted to full-time clinical education experiences. In order to offer these vital student learning opportunities, clinics and healthcare facilities must be willing to affiliate with physical therapy education programs and host students. This willingness has begun to decline with the mean number of affiliated clinical education sites reported by Commission on Accreditation in Physical Therapy Education (CAPTE) accredited DPT programs dropping from 581 in 2016-17 to 537 in 2018-19 and the number of CAPTE accredited DPT programs increasing from 241 in 2016 to 261 with 57 more in development as of December 2020.2 The most currently available data for CAPTE accredited PTA programs reports the mean number of affiliated clinical education sites as 135 sites in 2019-120 for 378 programs with 38 more in development as of December 2020. Recent literature has indicated this decline may be due to the difficulty for the clinician hosting a student to maintain productivity standards, a lack of support from clinic/facility administrators, inadequate staffing, space limitations, and decreased time for practice management duties while hosting a student. ${ }^{3-4}$ These barriers have the potential to cause clinics to question accepting students for clinical education experiences.

Much of the research over the past three decades investigating the impact on physical therapist productivity while hosting a student consistently concluded hosting a student has a positive impact on productivity. 1,3,5-11 The Dillon et al study of four-week clinical experiences occurring at five inpatient acute or inpatient rehab sites found an increase in the number of patients seen and units generated per day for clinicians hosting students. ${ }^{1}$ In the study by Pivko, et. al that used self-reported productivity logs from acute care, outpatient, acute rehab, and mixed/specialty care settings, it was found that experiences lasting six-weeks or longer showed no significant differences between the two baseline weeks when compared to weeks one and the final week of the experience. ${ }^{9}$ They recorded number of patients per hour, number of billable treatments per hour, number of initial evaluations per hour, and number of hours worked per day. They found a productivity increase in the number of patients treated per hour worked at week six that was significantly greater than the number of patients treated at baseline. The recent Apke, et al study found that the productivity of a clinician hosting a student was significantly increased for the outpatient orthopedic and inpatient rehab settings and approached significance for outpatient neuro settings, but was not significant in acute care settings when comparing clinicians hosting students to those in the same facilities not hosting a student. ${ }^{3}$ Past research has also focused solely on DPT students, with little to no data reporting the impact of hosting PTA students.

The issue of clinician productivity is not solely limited to the field of physical therapy. Similar studies in occupational therapy (OT) show there is no difference in clinical productivity with or without a student in inpatient rehabilitation, outpatient, and pediatric settings during a 12 week student experience using a 1:1 model, although it was suggested productivity with a student decreased with less years of experience. ${ }^{12}$ These findings were similar to Patterson's study over three years showing no difference in productivity in a psychiatric setting. ${ }^{13}$ More recent studies have been found in other health care professional literature from Australia, Europe and Canada. ${ }^{13-15}$ In dietetics, it has been shown in the Ash et al study that hosting students does not reduce the occasions of service and actually increases the project activities in both food service and public health, whereas in the Kämäräinen et al study involving both OT and dietetics, there was an increased length of service and trend in number of occasions of service when students were present. 7,14

The purpose of this study is to examine the impact on clinician productivity while hosting a student during a clinical experience. This study (a) investigates differences seen when hosting a DPT student or a PTA student; (b) compares differences seen influenced by which curricular stage the student is in for the clinical experience; and (c) investigates the impact on productivity while hosting a student across various clinic settings. It is hypothesized that the results of this study will disprove the common belief that clinician productivity is negatively impacted while hosting a student during a clinical experience.

\section{METHODS}

This study examined the productivity of clinicians who hosted DPT and PTA students from three Kansas metro academic institutions. This was a cross-sectional study with a survey to a selective purposively chosen sample by a quick, convenient, and cost-effective distribution method. Data looked at the week-to-week impact students had (a) whether they were DPT or PTA students, (b) when students were in different stages of a curriculum, (c) and when students were in different settings while they progressed through a clinical education experience. Using a similar definition for productivity as is found in PT productivity in clinical education literature, the data collection included the number of hours worked, number of patients seen, and number of units billed. ${ }^{1,3}$ These measures were chosen to allow for easier application across multiple practice settings. Varied reimbursement sources and 
site-specific measures make it challenging to compare actual productivity across settings. The data points selected measure productivity from a unit level. ${ }^{15}$

\section{Participants}

Clinicians who served as clinical instructors $(\mathrm{Cl})$ for participating students from 2 DPT programs and 1 PTA program during the study period were non-randomly targeted and invited once to participate in this study. Data was collected for a total time span of 18 months with data form University of Saint Mary DPT students collected from August 2016 to May 2017, data for University of Kansas Medical Center DPT students collected from January 2016 to July 2016, and data from Kansas City Kansas Community College PTA students collected from August 2015 to May 2017. Data from clinicians who had a different student the week prior to the first day of a student was excluded. There were no withdrawal/termination criteria. The purpose of the project was explained to all students, and those students participating in the study were oriented to the data collection form and data collection methods prior to going to the clinic and reminded periodically during their experience to ensure to complete the collection. For PTA and University of Kansas Medical Center DPT students, it was a required part of the clinical education course while for the University of Saint Mary DPT students it was an option provided for meeting a course project.

\section{Data Collections}

The data collection form was specifically designed for this study. The instrument collected daily hours worked, units billed, and number of patients treated. Baseline data for the $\mathrm{Cl}$ was gathered for the week immediately prior to the student's arrival as selfreported by the $\mathrm{Cl}$. The baseline timeframe was chosen to increase participation by limiting the burden of extended $\mathrm{Cl}$ reporting. This timeframe also allowed more clinician eligibility to participate in the study as many Cls often host multiple students per year and have limited time without a student. Daily data for every week while the student was on their clinical education experience was recorded. Each student was instructed to complete the form daily in collaboration with their $\mathrm{Cl}$. Students self-selected to record data either manually or electronically. Those who manually completed the form submitted either a scanned copy via email or a hard copy. Those who electronically completed the form did so through the university learning management system. Additional information recorded on each data collection form included the type of setting (outpatient, inpatient, other), the level of clinical education experience (initial, intermediate, final), the type of student (DPT, PTA), and the date ranges of the experience.

Institutional Review Board approval was obtained from respective institutions. All students participating in the study were oriented to the data collection form and data collection methods prior to going to the clinic and reminded periodically during their experience to ensure to complete the collection. Students explained the purpose of the project to clinical instructors at the beginning of the experience and disclosed all research study information with a written request by the investigator for participation. $\mathrm{Cl}$ and student consent to participate in the study was implied through submission of a completed data collection form. Completed data collection forms were returned to the authors as a component of their job roles as Directors of Clinical Education within each educational program for data analysis.

\section{Data Analysis}

Because of the variation in curriculums, it was decided for sub-analysis based on timing of the full-time clinical experience (FTCE) that initial experiences were considered the first FTCE, final FTCE was considered the very last FTCE and intermediate FTCEs were all FTCEs between the initial and final. Not enough entries were anticipated to show meaningful results in every setting and there was high variability in the specific types of setting between DPT and PTA students, so data was grouped into inpatient, outpatient, and other (home health, school system, mixed IP/OP) for analysis. University of Saint Mary DPT students completed a nine-week FTCE for all phases. University of Kansas Medical Center DPT students completed an eight-week FTCE for all phases. Kansas City Kansas PTA students completed a four-week beginning FTCE, a four-to-six week (average 5 week) intermediate FTCE, and a six-week FTCE for final experiences.

Data entry was double checked by an independent researcher to ensure accurate data transfer for manually submitted forms. Data collection form responses were coded for statistical analysis in Sigma Plot 14.0 (SyStat Software Inc. San Jose, CA). Exclusion criteria included $\mathrm{Cl}$ refusal to participate, more than two weeks of data not filled out, only one or two components of data recorded (i.e., hours, number of patients, units billed), and obvious signs that the student collected data on themselves rather than the $\mathrm{Cl}$-student team. If one to two days of data were missing (i.e., due to student/Cl illness, holidays, etc.), they were treated as missing data and the average was taken for the number of days for which there was data.

Productivity was analyzed by comparing the baseline productivity data for the clinical instructor with the productivity data for each week during the full-time clinical experience. Weekly averages for each variable (hours worked, units billed, number of patients 
treated) were calculated from daily data. A collective weekly average was calculated for the cohort's FTCE by averaging all the individual student weekly averages. The collective weekly averages were then compared over time (week to week) using one-way repeated measures ANOVA with $p<0.05$ for statistical significance. Independent samples t-tests, with $p<0.05$ for statistical significance, were used to examine differences between baseline averages and collective weekly averages while hosting students on FTCE. Sub-analysis using the above statistical analysis was completed for examination of the phase in curriculum (beginning, intermediate, final) and practice settings (outpatient, inpatient, other).

\section{RESULTS}

Surveys from 138 physical therapy students and 68 physical therapy assistant students were received. Nine PTA and four DPT student responses were excluded for a total of 59 PTA and 134 DPT student data sets used (Table 1). $100 \%$ of PTA students, $82.2 \%$ of University of Saint Mary DPT students, and $49.1 \%$ of University of Kansas Medical Center DPT students recruited submitted responses which encompasses both $\mathrm{Cl}$ and student participation. All students were in a 1:1 model of clinical education.

Table 1: DPT and PTA student completion by length and setting of FTCEs

\begin{tabular}{|l|l|l|}
\hline & PTA $(\mathrm{n}=59)$ & DPT ( $\mathrm{n}=134)$ \\
\hline Experience setting & & \\
Outpatient & 27 & 87 \\
Inpatient & 26 & 43 \\
Other & 6 & 4 \\
\hline $\begin{array}{l}\text { Length of experience } \\
\text { Initial }\end{array}$ & 9 (4 weeks) & $29(8-9$ weeks $)$ \\
Intermediate & 29 (4 or 6 weeks $)$ & $80(8-9$ weeks $)$ \\
Final & $21(6$ weeks $)$ & $25(8-9$ weeks $)$ \\
\hline
\end{tabular}

The number of entries from the students for each day of each week varied (Table 2). Baseline data was completed in $64.4 \%$ of PTA students and in $53.7 \%$ of DPT student data sets. Of PTA data sets, week one completion was $94.9 \%$ and week 6 was $94.7 \%$ completion while all other remaining weeks were $100 \%$ complete. Of the DPT data sets, weeks $1-2$ were $99.3 \%$ complete. Weeks 3-5 were $100 \%$ complete. Week 6 was $98.5 \%$ complete while weeks $7-8$ were $97.0 \%$ complete and week 9 was $88.0 \%$ complete.

Table 2: Average completion rate per week

\begin{tabular}{|l|l|l|l|l|}
\hline Daily hours worked & $\mathrm{N}=$ & $\%$ completion & Mean value & Std. Dev. \\
\hline PTA baseline & 57 & $67 \%$ & 8.16 & 1.17 \\
\hline PTA week 1 & 59 & $95 \%$ & 8.05 & 1.10 \\
\hline PTA week 2 & 59 & $100 \%$ & 8.11 & 1.01 \\
\hline PTA week 3 & 59 & $100 \%$ & 8.18 & 1.12 \\
\hline PTA week 4 & 59 & $100 \%$ & 8.11 & 1.01 \\
\hline PTA week 5 & 38 & $100 \%$ & 8.20 & 1.03 \\
\hline PTA week 6 & 38 & $95 \%$ & 8.31 & 1.27 \\
\hline DPT baseline & 134 & $54 \%$ & 8.48 & 0.97 \\
\hline DPT week 1 & 134 & $99 \%$ & 8.42 & 0.79 \\
\hline DPT week 2 & 134 & $99 \%$ & 8.42 & 1.00 \\
\hline DPT week 3 & 134 & $100 \%$ & 8.41 & 0.94 \\
\hline DPT week 4 & 134 & $100 \%$ & 8.43 & 0.98 \\
\hline DPT week 5 & 134 & $100 \%$ & 9.39 & 1.00 \\
\hline DPT week 6 & 134 & $99 \%$ & 8.31 & 0.93 \\
\hline DPT week 7 & 134 & $97 \%$ & 8.41 & 0.98 \\
\hline DPT week 8 & 134 & $97 \%$ & 8.34 & 0.98 \\
\hline DPT week 9 & 25 & $88 \%$ & 8.29 & 0.67 \\
\hline \# daily patients & & & & \\
\hline PTA baseline & 57 & $67 \%$ & 7.72 & 1.59 \\
\hline
\end{tabular}




\begin{tabular}{|l|l|l|l|l|}
\hline PTA week 1 & 59 & $95 \%$ & 7.12 & 2.21 \\
\hline PTA week 2 & 59 & $100 \%$ & 7.43 & 2.25 \\
\hline PTA week 3 & 59 & $100 \%$ & 7.47 & 2.16 \\
\hline PTA week 4 & 59 & $100 \%$ & 7.43 & 1.89 \\
\hline PTA week 5 & 38 & $100 \%$ & 7.66 & 2.07 \\
\hline PTA week 6 & 38 & $95 \%$ & 7.67 & 2.20 \\
\hline DPT baseline & 134 & $52 \%$ & 9.22 & 2.88 \\
\hline DPT week 1 & 134 & $99 \%$ & 8.43 & 2.97 \\
\hline DPT week 2 & 134 & $99 \%$ & 8.76 & 2.94 \\
\hline DPT week 3 & 134 & $100 \%$ & 9.04 & 3.53 \\
\hline DPT week 4 & 134 & $100 \%$ & 9.28 & 3.27 \\
\hline DPT week 5 & 134 & $100 \%$ & 9.47 & 3.53 \\
\hline DPT week 6 & 134 & $99 \%$ & 9.26 & 3.38 \\
\hline DPT week 7 & 134 & $97 \%$ & 9.42 & 3.32 \\
\hline DPT week 8 & 134 & $97 \%$ & 9.40 & 3.49 \\
\hline DPT week 9 & 25 & $88 \%$ & 8.09 & 2.89 \\
\hline \# daily units billed & & & & \\
\hline PTA baseline & 57 & $67 \%$ & 26.21 & 8.68 \\
\hline PTA week 1 & 59 & $95 \%$ & 24.20 & 9.40 \\
\hline PTA week 2 & 59 & $100 \%$ & 25.04 & 9.09 \\
\hline PTA week 3 & 59 & $100 \%$ & 24.69 & 8.59 \\
\hline PTA week 4 & 59 & $100 \%$ & 25.35 & 8.55 \\
\hline PTA week 5 & 38 & $100 \%$ & 26.49 & 9.51 \\
\hline PTA week 6 & 38 & $95 \%$ & 26.78 & 8.62 \\
\hline DPT baseline & 134 & $54 \%$ & 30.16 & 11.72 \\
\hline DPT week 1 & 134 & $99 \%$ & 28.51 & 12.88 \\
\hline DPT week 2 & 134 & $99 \%$ & 29.65 & 13.69 \\
\hline DPT week 3 & 134 & $100 \%$ & 31.18 & 15.72 \\
\hline DPT week 4 & 134 & $100 \%$ & 31.82 & 14.30 \\
\hline DPT week 5 & 134 & $100 \%$ & 32.51 & 15.25 \\
\hline DPT week 6 & 134 & $99 \%$ & 32.42 & 15.73 \\
\hline DPT week 7 & 134 & $97 \%$ & 32.41 & 14.60 \\
\hline DPT week 8 & 134 & $97 \%$ & 32.37 & 15.60 \\
\hline DPT week 9 & 25 & $88 \%$ & 25.93 & 6.95 \\
\hline & & & & \\
\hline & & & \\
\hline
\end{tabular}

Of the overall PTA surveys analyzed, $15.2 \%$ were initial, $49.2 \%$ were intermediate, and $35.6 \%$ were final full-time clinical experiences (FTCE). Forty-six (45.8\%) took place in outpatient settings, $44.1 \%$ were in inpatient settings and $10.1 \%$ were in other types of settings including mixed IP-OP settings and home health. Of the DPT surveys analyzed, $21.6 \%$ were initial, $59.7 \%$ were intermediate and $18.7 \%$ were final FTCE. Sixty-five (64.9\%) were outpatient, $32.1 \%$ were inpatient and $3.0 \%$ were other types of settings including school system, home health and mixed settings.

\section{Type of Student (DPT, PTA) \\ Hours Worked}

The baseline PTA Cl hours worked was 8.16 and interestingly, week 1 of hosting a student was the lowest average of hours worked at 8.05 and highest was during week 6 , the final week, with 8.31 hours (figure $1 \mathrm{~A}$ ). There was very little variance week to week except for during the last week which added 10 minutes daily. No significant differences were seen across the entire time of the FTCE or between baseline (8.16) and the collective average daily Cl hours worked (8.15) while hosting a PTA student.

The baseline DPT Cl hours worked was 8.48 . Hours worked slightly decreased throughout the FTCE with the highest number of hours worked of 8.42 occurring during weeks $2-3$ and lowest of 8.29 occurring during week 9 (figure $1 \mathrm{~A}$ ). No significant differences were seen across the entire time of the FTCE or between the baseline (8.48) and the collective average daily $\mathrm{Cl}$ hours worked while hosting a DPT student (8.39). 
A

Overall Hours Worked

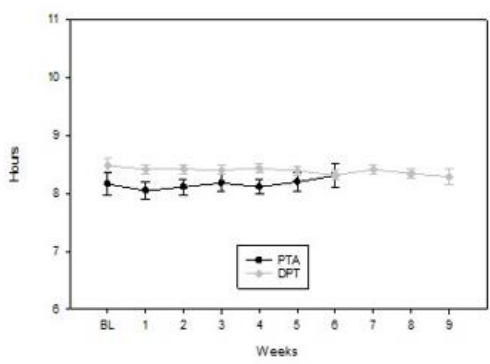

Overall Number Daily Patients Seen

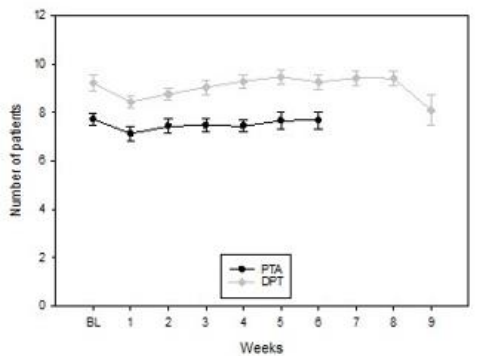

C

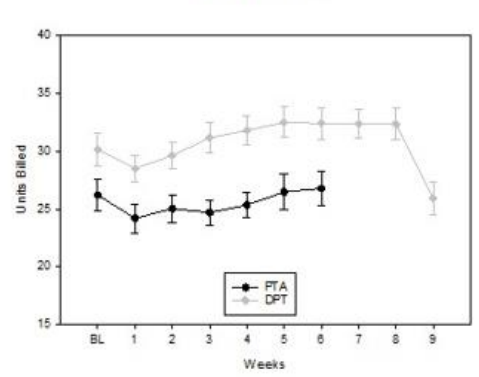

Figure 1. Type of Student (DPT, PTA)

A. No significant differences were found in any of the average daily hours the $\mathrm{Cl}$ worked while hosting a DPT or PTA student.

B. PTA: The overall number of daily patients seen significantly changed over time $(p=0.027)$. DPT: The overall number of daily patients seen significantly changed over time $(p<0.001)$ except for the last week which drops off. Week 5 levels were significantly higher than baseline.

C. PTA: No significant differences were found in any of the $\mathrm{Cl}$ units billed. DPT: Significant changes over time were observed overall $(p<0.001)$.

Bars represent standard error. $\mathrm{BL}=$ baseline.

\section{Number of Daily Patients Seen}

The mean PTA Cl baseline number of daily patients seen was 7.72 (figure 1B). This number changed over time $(p=0.027)$ with a significant drop during week one to 7.12 patients and moving back to near baseline levels by week 5 at 7.66 patients. However, there was no significant difference between baseline (7.72) and the collective average number of patients seen daily (7.50).

The mean DPT Cl baseline number of daily patients seen was 9.22 (figure 1B). This also demonstrated a significant change over time ( $p<0.001$ ), first with a significant loss during week one to 8.43 patients and then exceeding baseline by week 4 (9.28) while reaching a high of 9.47 average daily patients for the week in week 5 . The number of patients in weeks 5-8 were significantly higher than during weeks 1-2. Week 9 showed a drop to 8.09 average daily patients. There was no significant difference between the number of patients seen during the baseline week (9.22) and the collective average number of patients seen daily across the entire FTCE (9.01).

\section{Units Billed}

The $\mathrm{Cl}$ baseline units billed for PTA students was 26.2 units. The units billed dropped to 24.2 in week one reaching baseline levels at week 5 (26.5) but maximized week 6 at 26.8 units (figure 1C). Change over time for units billed was not statistically significant nor was the difference between baseline and the collective weekly average during the entire FTCE (25.3).

For Cls hosting DPT students, the units billed had significant changes over the FTCE $(p<0.001)$ (figure $1 \mathrm{C})$. The $\mathrm{Cl}$ average daily baseline was 30.16 units and demonstrated a significant dip the first week to 28.51 units recovering by week 3 (31.18). This recovery by week 3 allowed weeks $4-8$ to be significantly higher than weeks 1-2. Similar to prior results, there was a decline to 25.93 average daily units billed in the final week. When comparing baseline (30.16) to averaged collective daily units billed (30.75), there were no significant differences. It appears that there was, on average, almost one more daily unit billed over the course of 9 weeks which may demonstrate a financial gain even though slightly less patients were seen.

\section{Level of Clinical Education Experience (initial, intermediate, final) \\ Hours Worked}

When the data were grouped by the timing of the FTCE, for Cls hosting PTA students, a slight dip of 10 minutes daily was observed during weeks 1-2 from the initial FTCE baseline average daily hours worked of 8.39 and returned to baseline by week 3 (figure $2 \mathrm{~A})$. During intermediate experiences, all weeks of hosting a student resulted in lower average $\mathrm{Cl}$ hours worked compared to baseline. An initial dip from 7.94 baseline hours worked to 7.62 hours was observed at week 1 . The highest hours worked while hosting a PTA student, which remained below baseline, occurred during week 3 at 7.91 hours (figure 2A). For final experiences, the baseline hours worked were 8.46 with very little variance across time until the final week (week six) which increased to 8.75 hours (figure 2A). There were no significant differences across the entire time of the FTCE for any of the groups for hours worked, nor in comparing baseline hours to the collective average daily $\mathrm{Cl}$ hours worked during the FTCE. 
For Cls hosting DPT students during initial FTCE, baseline hours worked was 8.63 which remained steady slightly dropping only during week 4-5. The lowest hours worked was reached during their final week of the initial FTCE at 8.36 hours (figure 2A). Intermediate FTCE baseline hours worked was 8.34 and remained steady again dropping down towards the end of the FTCE to a low of 7.98 hours during the final week (figure 2A). A different trend was seen for final FTCE as DPT CI baseline hours worked was 8.50 and remained very steady until the final week then jumping to 9.00 hours (figure $2 \mathrm{~A}$ ). None of these differences were statistically significant nor were comparisons of average baseline hours $(8.63,8.34,8.50)$ to collective daily average hours $(8.57$, $8.30,8.47$ ) for each type of FTCE.

A

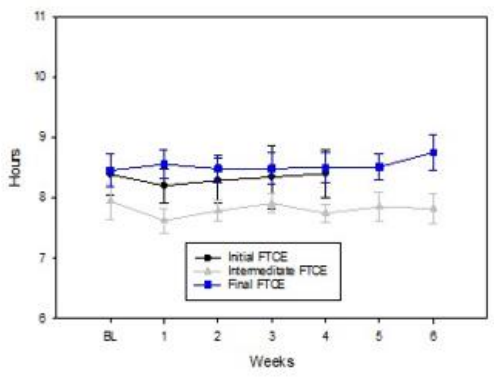

DPT CI Hours Worked

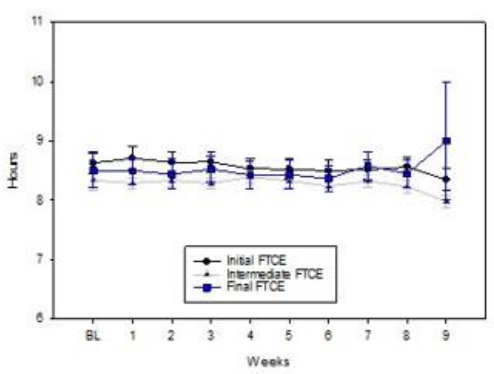

B

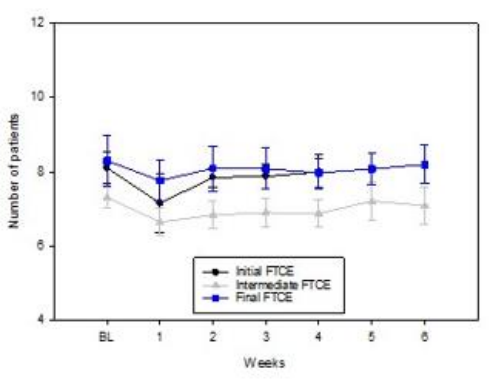

DPT CI Daily Patients Seen

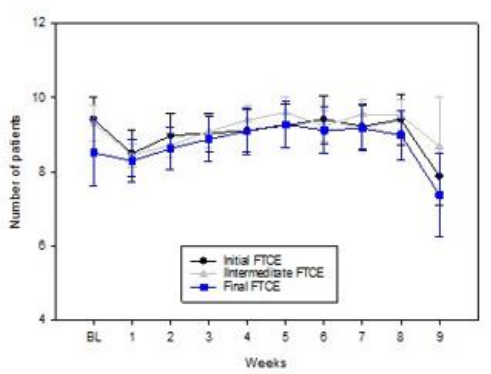

C

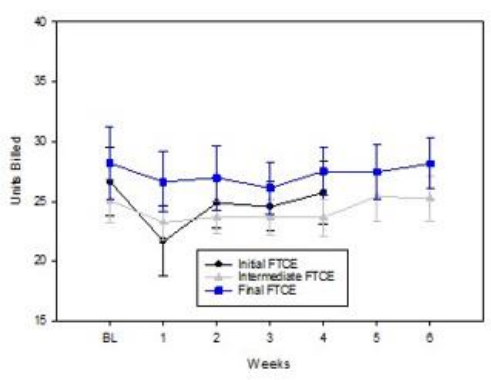

DPT CIUnits Billed

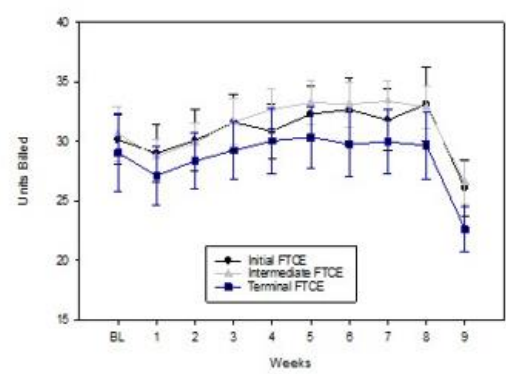

Figure 2. Level of Clinical Education Experience (initial, intermediate, final)

A. No significant differences were found in any of the average daily hours the $\mathrm{Cl}$ worked while hosting a DPT or PTA student.

B. PTA: No significant changes were seen likely due to the larger standard deviation. DPT: There was a significant difference over time during intermediate clinical experiences $(p<0.001)$ and trends towards significance in both initial and final FTCEs.

C. PTA: The only significant change observed over time was during initial FTCE $(p=0.04)$. DPT: Significant changes over time were observed in initial $(p<0.001)$, intermediate $(p<0.001)$, and final FTCEs $(p=0.021)$.

Bars represent standard error. $\mathrm{BL}=$ baseline.

\section{Number of Daily Patients Seen}

During the initial PTA FTCE, the average daily number of patients seen at baseline was 8.10 , which dipped the first week to 7.15 , then increased week 2 to 7.84 ending at 7.99 week 4 (figure 2B). During the intermediate FTCE, the baseline number of patients seen by PTA Cls was 7.31, dipping to the lowest of 6.64 during week one and the highest of 7.21 at week 5 (figure 2B). During the final FTCE, the baseline number of patients seen daily was 8.29 , dropping to the lowest at week one (7.76), achieving the highest week 2 (8.09), and remaining steady throughout the remainder of the FTCE weeks (figure 2B). There were no statistical differences in initial, intermediate, and final groups over the entire time of the FTCE nor in the comparison of baseline (7.72) to the collective average of all weeks (7.44).

For DPT students on their initial FTCE, the average number of patients seen dipped week one to 8.5 from baseline of 9.42 with steady recovery to baseline reached in week 6 (figure $2 \mathrm{~B}$ ). The final week showed a decline to 7.88 averaged daily patients. This difference was nearing significance $(p=0.077)$. However, when comparing the baseline $(9.42)$ to the collective average number of patients seen daily (9.06) there were no differences. During intermediate FTCE, the DPT student Cls did show a significant change over time (figure 2B). Baseline average daily patients seen was 9.31. This average value significantly dipped week one to 8.45 with recovery to baseline levels by week $4(9.40)$ reaching a high of 9.60 at week 5 . Weeks $5-8$ were significantly higher than 
weeks 1-2. The collective average number of patients seen daily for all weeks (9.20) was not statistically different from baseline (9.31). During final FTCE, DPT Cls saw 8.52 patients on average at baseline (figure 2B). Week one dipped to 8.30 patients and recovered to baseline levels by week two (8.63) reaching a high of 9.3 patients by week 5 . Number of patients seen remained above baseline weeks $2-8$ dipping down to 7.38 the final week. There was a trend toward significance $(p=0.113)$. Again, the difference between baseline (8.52) and the collective average of daily patients seen each week (8.92) was insignificant.

\section{Units Billed}

There was a significant change over time during the initial FTCE that were 4 weeks in length $(p=0.044)$. Baseline was 26.7 daily units dropping to 21.7 the first week, gradually increasing to a high of 25.7 daily units the final week (figure $2 \mathrm{C}$ ). Intermediate baseline average daily units billed was 25.1 showing a 2 unit drop the first week to 23.2 but returning to baseline levels week 5 (25.4) and remaining stable the final week (figure $2 \mathrm{C}$ ). Final baseline levels were 28.2 with a drop to 26.7 week one moving back towards baseline levels by week 6 (28.2) (figure 2C). Neither intermediate nor final changes over time were significant. None of the comparisons of baseline to collective weekly averages were significant.

When accounting for the timing of the FTCE that Cls hosted DPT students, the initial, intermediate and final FTCE (figure 2C) followed similar significant changes and patterns as seen in the results of changes that occurred over the entire time of the FTCE in daily units billed ( $p<0.001, p<0.001$ and $p=0.021$ respectively). When comparing baseline to averaged collective units billed, there were no significant differences in initial $(30.15,31.13)$, intermediate $(30.64,31.89)$ or final FTCE $(29.0,29.27)$. There was also no change over time in units billed for Cls hosting DPT students in other settings.

\section{Type of Setting (outpatient, inpatient, other) Hours Worked}

For PTA students, the baseline $\mathrm{Cl}$ hours worked in the outpatient (OP) setting was 8.29 , inpatient (IP) settings was 7.1 , and other settings was 6.65 hours (figure 3A). OP PTA Cl hours dipped to 7.74 the first week and did not reach baseline hours until week 5 . There was a trend towards working less hours when students were present although not significant $(p=0.119)$. IP hours were lowest (6.78) at week one and highest at week 4 (7.36) with a significant difference $(P=0.031)$ over time compared to baseline being higher in weeks 5 and 6 . Other settings were also lowest week one at 5.52 and remained lower, not reaching the baseline level of 6.65 through the remainder of the FTCE, although this difference was not significant.

For DPT students, the baseline $\mathrm{Cl}$ hours worked in OP was 8.73 while that of the IP $\mathrm{Cl}$ was 8.14 hours (figure $3 \mathrm{~A}$ ). In OP, the average daily $\mathrm{Cl}$ hours worked remained lower than the baseline progressively lowering throughout with the lowest being 8.31 hours in week $9(\mathrm{p}=0.138)$ although not a statistically significant difference. The IP hours remained near baseline levels until the last week shifting slightly up to 8.26 hours. The baseline $\mathrm{Cl}$ hours worked for other settings was 6.5 and increased to 7.3 week one with a high of 7.93 hours during weeks 6-7. For Cls hosting DPT student, there were no significant differences in the average daily hours worked over the entire time of the FTCE in any setting, nor when comparing baseline to collective averages of daily hours worked when the student was present.

\section{Number of Daily Patients Seen}

For PTA students on OP FTCE, average daily number of patients seen at baseline was 8.29 hitting a low of 7.72 patients in week 4 before returning to baseline levels at week 5 and then surpassing baseline levels to 8.34 in week 6 , which was trending toward significance $(p=0.119)$ (figure $3 B)$. For the IP settings, there was a significant positive change over time $(p=0.031)$. The average number of patients treated at baseline was 7.10 (figure 3B). It dipped the first week to 6.78 patients but surpassed baseline levels weeks 2-5 maximizing at week 4 with 7.36 patients seen on average daily before returning to baseline levels at week 6 (7.15). Cls hosting PTA students in other settings showed no significant differences and had average baseline number of patients seen of 6.65 dropping to the lowest of 5.52 week one and peaking in week 4 at 6.31 .

For DPT students in OP settings, the baseline daily average was 9.92 patients showing a decrease week one to 8.91 with recovery to baseline levels during week 4 (9.91) and exceeding baseline in weeks 5-8 before falling the final week to 8.15 (figure 3B). This was a significant change over time $(p<0.001)$ showing the same patterns seen overall. For DPT students in IP FTCE, there again was a significant change over time $(p=0.041)$ in the average daily number of patients seen with a baseline of 8.16 dipping the first week to 7.42 recovering back to baseline levels week $5(8.13)$ with a high of 8.40 week 7 before again falling off the final week to 7.86 daily patients (figure $3 \mathrm{~B}$ ). However, when comparing baseline to collective average daily patients seen each week, there were no differences in $\mathrm{OP}(9.92,9.69)$ or IP $(8.15,7.99)$ daily average number of patients seen. For DPT students in other settings, there were no significant changes over time. 
A

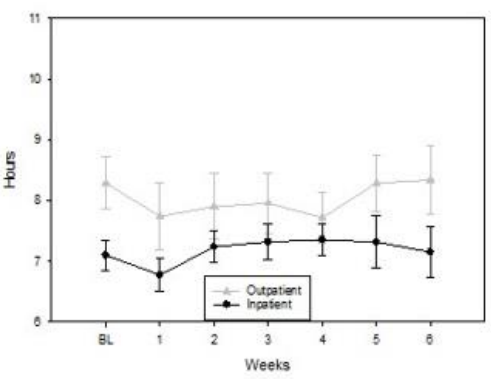

DPT CI Hours Worked

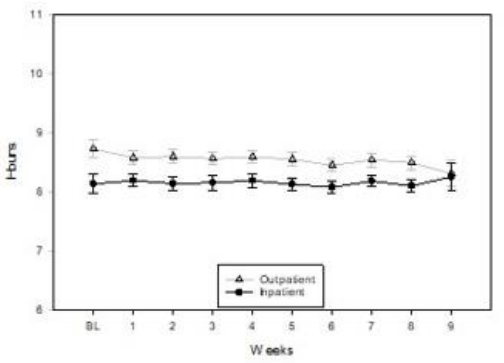

B

PTA CI Daily Patients Seen

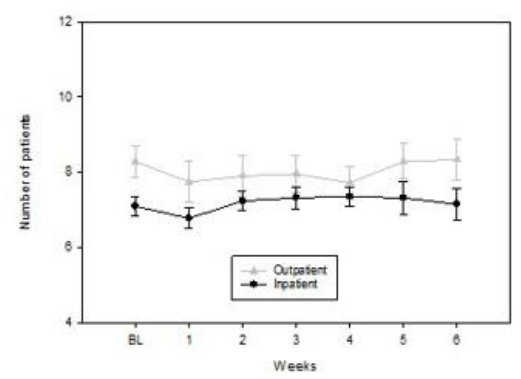

DPT CI Daily Patients Seen

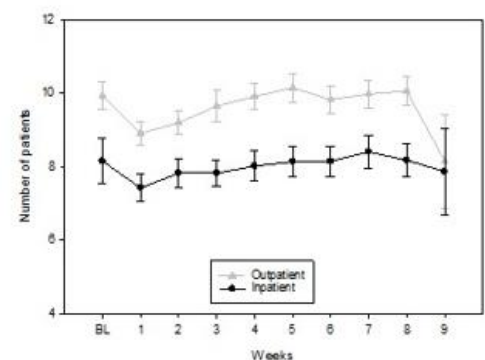

C PTACI Units Bllled

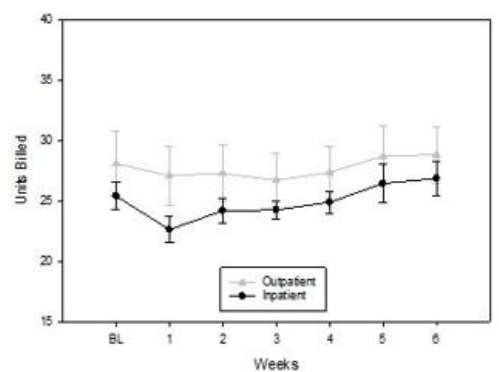

DPT CIUnits Bllled

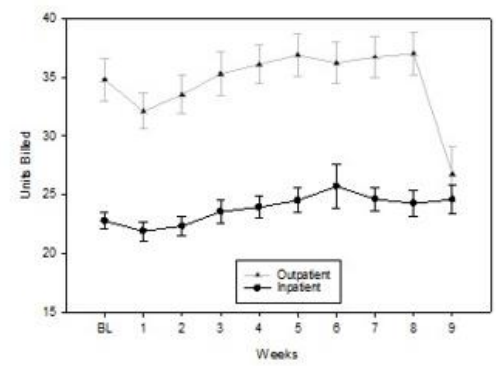

Figure 3. Type of Setting (outpatient, inpatient, other)

A. PTA: The only significant change in time $(p=0.03)$ was seen during inpatient settings with a reduction of hours worked the first week of hosting a student, rebounding the second week of the clinical experience. DPT: No significant differences were found in any of the average daily hours the $\mathrm{Cl}$ worked.

B. PTA: There was a significant change over time with clinician productivity in inpatient settings $(p=0.031)$ and a trend in outpatient settings $(p=0.11)$. DPT: There were significant differences in both inpatient $(p=0.04)$ and outpatient settings $(\mathrm{p}<0.001)$.

C. PTA: The only significant change observed over time was in inpatient settings $(p=0.002)$. DPT: Significant changes over time were observed in FTCEs in outpatient $(p<0.001)$ and inpatient $(p=0.003)$ settings.

Bars represent standard error. $\mathrm{BL}=$ baseline.

\section{Units Billed}

There was no significant change over time for Cls hosting PTA students for the outpatient or other settings but there was a significant difference in the inpatient setting $(p=0.002)$ (figure $3 C$ ). The baseline average daily units billed was 25.4 dropping the first week to 22.6 with steady recovery surpassing baseline levels by week $5(26.4)$ reaching a high of 26.9 week 6 . None of the comparisons of baseline to collective weekly averages were significant.

In OP settings, the DPT student $\mathrm{Cl}$ billed 34.82 average daily units at baseline (figure $3 \mathrm{C}$ ). A decline to 32.12 units was observed in the first week but recovered to baseline (37.01) by week 8 before falling the final week to 26.78 . This was a significant change over the entire time of the FTCE when comparing week to week $(p<0.001)$. When comparing the baseline (34.82) to the collective average daily units billed of 35.33 , there was no significant difference. The IP setting $\mathrm{Cl}$ average daily units billed also showed a significant change over the entire time of the FTCE $(p=0.003)$ but had a different pattern than previously seen (figure $3 C)$. The baseline average daily units billed started at 22.79, declined week one to 21.91, exceeded baseline units by week 3 (23.57), steadily climbed to week 6 (25.72), and maintained higher than baseline levels the remainder of the weeks without any final week drop off.

\section{DISCUSSION}

The impact of clinician productivity in direct patient care while hosting a student for a clinical experience was examined to disprove the common belief that clinician productivity is negatively impacted while hosting a student during a clinical experience. Clinicians hosting DPT and PTA students from three greater Kansas metro academic institutions were studied in this project. The research hypothesis that overall clinician productivity, as calculated using the number of hours worked, patients seen, and units billed, would 
not be negatively changed by hosting a student was supported in this study. Evidence also showed that while productivity may initially decline during the first two to three weeks of a student clinical experience, it rebounds and matches or increases beyond average baseline clinician productivity levels when not hosting a student across the remaining weeks of the clinical experience. The bottom line comparison of the weekly collective average of the FTCE to baseline levels without students showed no significance even though there were significant changes over time.

This study supports the literature available demonstrating that hosting a student has a positive impact on clinician productivity, specific to the increase in number of patients seen, increase in number of units billed, and/or increase in overall productivity of the hosting clinician. $1,3,5,6,9,10$ Overall, the general trend observed throughout all analyses in this study demonstrates that hosting a student causes a small dip from baseline data to week 1 data, a recovery equal with baseline data by week 4 , an increase beyond baseline data during weeks 5-7, and then a return to baseline data during the final week of the experience. The initial dip seen between the baseline data and the week 1 data is thought to occur as orientation of the student to the clinic and operations of the clinic occur largely during the first week of a full-time clinical experience (FTCE). The recovery to baseline by week 4 and continued increase identified in the later weeks of the experience support the overall FTCE being net neutral therefore further supporting that hosting a student for a clinical experience does not negatively impact a clinician's productivity.

This study is the first of its kind to investigate the differences seen related to the timing of the FTCE as well as taking PTA students or DPT students. This study differentiated between students on an initial, intermediate, or final FTCE. No significant results were found related to hosting a PTA student when analyzed in relation to the timing of the FTCE for the variables of hours worked, number of daily patients seen, or units billed. The results when hosting a DPT student showed a significant increase in the number of patients seen during intermediate FTCE, with the number of patients seen during initial and final FTCE trending toward significance. Significance most likely was not achieved for data related to initial and final FTCE due to a larger number of intermediate FTCE data points compared to initial or final during this collection period. Continuing research in this area with equal subject numbers in each FTCE would further strengthen findings. The results from this study also show that DPT students during an initial FTCE require more time to recover to baseline for number of patients seen (recovering by week 6), while DPT students during an intermediate FTCE recover to baseline by week 4, and DPT students during a final FTCE recover to baseline by week 2. The most obvious reason for these results is that students are anticipated to orient more quickly to settings as they gain more clinical experience. The dip seen in the data during the final week of the FTCE, regardless of its timing, is thought to be due to the offloading of patients from the DPT student in preparation of their departure from the clinic and completion of requirements such projects and/or in-services, and documentation and review of student performance assessments.

This study also differentiated the impact of the clinic setting on the number of patients seen and units billed while hosting a student. Again, no significant results were found related to hosting a PTA student when analyzed in relation to clinic setting. The data showed for clinicians hosting DPT students in outpatient (OP) settings, there was a significant increase in the average daily number of units billed when comparing values weekly across the length of the FTCE. Similarly, clinicians hosting DPT students in inpatient (IP) settings saw a significant increase in the average daily number of units billed when comparing values weekly, but in IP settings, the higher than baseline levels remained throughout the weeks of the FTCE without any final week drop off as was seen with the OP settings. No significant differences were observed when hosting DPT students in other settings. The ability of the IP settings to maintain the gains seen are most likely due to the decreased ability of inpatient clinicians to control their daily schedules in the way outpatient clinicians can and therefore less offloading in preparation for the student's departure. IP settings maintain units billed in the final week, while OP settings final week dips like other areas. Even though the number of patients declined slightly when compared to baseline, one more unit was billed weekly. This suggests treatment pattern changes because while there were fewer patients seen, more units were billed weekly.

\section{Limitations}

One limitation to this study was the highly varied number of daily entries on a week-to-week basis. The DPT students only had a $100 \%$ daily response rate in weeks 4 and 5 . The PTA students only had a $100 \%$ daily response rate in the number of patients treated during weeks 2,3 , and 4 . For both groups of students, the number of baseline entries were significantly lower than the number of students represented in each group. This could have an impact on comparing the baseline numbers to the weeks with students. Future studies should make sure the number of entries is consistent from baseline to the end of the student's FTCE. Another limitation is that there were not enough entries in each setting for each profession to show significant results. The most entries for the DPT students were outpatient and acute care and for PTA students were skilled nursing and outpatient settings. Variability in supervision of student practice where some Cls may progress their students more quickly than other Cls was not tracked and therefore a limitation. Future studies could investigate individual settings like sub-acute rehabilitation, home health, 
and school-based systems as initial results show there may be some variation that is setting dependent. This study did not capture other definitions of productivity like efficiency. This study does not consider the negative cost impact of orientation, compliance training/tracking, or other added costs, nor the positive cost savings of recruitment and marketing if they hire a student.

Another limitation to this study relates to accuracy of student reporting during the data collection period. Possible discrepancies between activities in the clinic compared to what was recorded on the data sheet may have occurred. Similarly, any time delay between clinic activity and student recording may also have impacted accuracy of data due to recall bias.

\section{CONCLUSION}

This study demonstrated that a clinic taking students during their FTCE does not significantly decrease productivity. In some cases, it increased the productivity, which could potentially lead to more financial gains for the clinic at that time. Clinics can use this data to have a better idea if they want to accept students during FTCE and how they want to structure their clinical education program. This study demonstrates that return on investment seems to occur during the latter half of longer term FTCE. This evidence may also allow Cls to recognize they have more time to devote to other practice management duties or project activities while supervising the student since the fear of decreased productivity, specifically units billed, was not shown to be substantiated. Increase in FTCE hours during the final week should be expected as Cls have to absorb the caseload seen by DPT students. The results of this study may also be used in academic program's considerations when determining length and placement of FTCE within the curriculum.

\section{REFERENCES}

1. Dillon LS, Tomaka JW, Chriss CE, Gutierrez CP, Hairston JM. The effect of student clinical experiences on clinician productivity. J Allied Health. 2003;32(4):261-5.

2. Commission on Accreditation in Physical Therapy. 2018-2019 Aggregate Program Data Alexandria, VA2019 [Available from: http://www.capteonline.org/uploadedFiles/CAPTEorg/About_CAPTE/Resources/Aggregate_Program_Data/Aggregate ProgramData_PTPrograms.pdf.

3. Apke TL, Whalen M, Buford J. Effects of Student Physical Therapists on Clinical Instructor Productivity Across Settings in an Academic Medical Center. Phys Ther. 2020 February; 100(2): 209-216.

4. Rapport MJ, Kelly MK, Hankin TR, Rodriguez JW, Tomlinson SS. A Shared Vision for Clinical Education: The YearLong Internship. Journal of Physical Therapy Education (American Physical Therapy Association, Education Section). 2014;28(Supp 1):22-9.

5. Lopopolo RB. Financial model to determine the effect of clinical education programs on physical therapy departments. Phys Ther. 1984;64(9):1396-402.

6. Ladyshewsky RK, Barrie SC, Drake VM. A comparison of productivity and learning outcome in individual and cooperative physical therapy clinical education models. Phys Ther. 1998;78(12):1288-98; discussion 99-301.

7. Rodger S, Webb G, Devitt L, Gilbert J, Wrightson P, McMeeken J. A clinical education and practice placements in the allied health professions: an international perspective. J Allied Health. 2008;37(1):53-62.

8. Davies R, Hanna E, Cott C. 'They put you on your toes': Physical Therapists' Perceived Benefits from and Barriers to Supervising Students in the Clinical Setting. Physiother Can. 2011;63(2):224-33.

9. Pivko SE, Abbruzzese LD, Duttaroy P, Hansen RL, Ryans K. Effect of Physical Therapy Students' Clinical Experiences on Clinician Productivity. J Allied Health. 2016;45(1):33-40.

10. Graham CL, Catlin PA, Morgan J, Martin E. Comparison of 1-day-per-week, 1-week, and 5-week clinical education experiences. J Phys Ther Educ. 1991;5(1):18-23.

11. Rindflesch AB, Dunfee HJ, Cieslak KR, Eischen SL, Trenary T, Calley DQ, et al. Collaborative model of clinical education in physical and occupational therapy at the Mayo Clinic. J Allied Health. 2009;38(3):132-42.

12. Ozelie R, Janow J, Kreutz C, Mulry MK, Penkala A. Supervision of Occupational Therapy Level II Fieldwork Students: Impact on and Predictors of Clinician Productivity. Am J Occup Ther. 2015;69(1):6901260010p1-7.

13. Paterson ML. Clinician productivity with and without students. Occup Ther J Res. 1997;17(1):48-54.

14. Ash S, Martin EK, Rodger S, Clark M, Graves N. Student and supervisor productivity change during nutrition and dietetic practice placements: A cohort study. Nutr Diet. 2015;72(2):163-9.

15. Kämäräinen VJ, Peltokorpi A, .Torkki P, Tallbacka K. Measuring health care productivity-from unit to system level. International Journal of HealthCare Quality Assurance 2016; 29(3):288-299. 